\title{
Laser Ablation of Drosophila Embryonic Motoneurons Causes Ectopic Innervation of Target Muscle Fibers
}

\author{
Te Ning Chang ${ }^{2}$ and Haig Keshishian 1 \\ 1/nterdepartmental Neuroscience Program, and ${ }^{2}$ Department of Biology, Yale University, \\ New Haven, Connecticut 06520-8103
}

We have tested the effects of neuromuscular denervation in Drosophila by laser-ablating the RP motoneurons in intact embryos before synaptogenesis. We examined the consequences of this ablation on local synaptic connectivity in both 1st and 3rd instar larvae. We find that the partial or complete loss of native innervation correlates with the appearance of alternate inputs from neighboring motor endings and axons. These collateral inputs are found at ectopic sites on the denervated target muscle fibers. The foreign motor endings are electrophysiologically functional and are observed on the denervated muscle fibers by the 1st instar larval stage. Our data are con- sistent with the existence of a local signal from the target environment, which is regulated by innervation and influences synaptic connectivity. Our results show that, despite the stereotypy of Drosophila neuromuscular connections, denervation can induce local changes in connectivity in wild-type Drosophila, suggesting that mechanisms of synaptic plasticity may also be involved in normal Drosophila neuromuscular development.

Key words: Drosophila; neuromuscular junction; motoneuron; plasticity; sprouting; collateral innervation; denervation; synaptogenesis; connectivity; muscle atrophy
In many systems, the development of synaptic connections is thought to involve multiple mechanisms, including molecular recognition and activity-dependent refinement (for review, see Goodman and Shatz, 1993). In seeking out their synaptic targets, growth cones can exhibit both attractive and repulsive responses to fixed or diffusible molecules (Landmesser, 1994; Kennedy et al., 1995). Furthermore, the initial projections and contacts made by growing axons may undergo alteration or refinement, changes that often involve electrical activity (for review, see Shatz, 1990). The neuromuscular junctions of Drosophila embryos and larvae are ideally suited for testing the roles of both cell recognition and synaptic plasticity in the establishment of correct synaptic connections. This system has been characterized extensively at both the cellular and the molecular level (Keshishian et al., 1996). Each abdominal hemisegment consists of 30 muscle fibers, innervated by $\sim 40$ motoneurons, in a segmentally repeated pattern. Many of the motoneurons are individually identifiable cells, allowing for the analysis of synaptic development with single-cell resolution (Halpern et al., 1991; Sink and Whitington, 1991a,b; Broadie and Bate, 1993a) (for review, see Keshishian et al., 1996). In addition, it is also possible to study the long-term effects of embryonic manipulations during the larval stages. The larval motor endings can often be differentiated as specific motoneurons on the basis of ending morphology (Cash et al., 1992; Keshishian et al., 1993; Kurdyak et al., 1994).

There is good evidence that during embryonic development Drosophila motoneurons identify their muscle fiber targets using molecular cues. Preference for specific muscle fibers was demon-

Received May 14, 1996; revised June 21, 1996; accepted June 25, 1996

This work was supported by grants from the National Science Foundation, National Institutes of Health, and NASA to H.K. We thank Dr. Robert Wyman, Dr. Robert Kalb, Dr. Jill Jarecki, Marc Halfon, and the members of the Keshishian lab for helpful comments, and Dr. Corey Goodman for reagents.

Correspondence should be addressed to Haig Keshishian, Department of Biology, Yale University, P.O. Box 208103, New Haven, CT 06520-8103.

Copyright (C) 1996 Society for Neuroscience $0270-6474 / 96 / 165715-12 \$ 05.00 / 0$ strated in experiments in which the number and patterns of fibers were altered using either genetic or microsurgical methods (Sink and Whitington, 1991c; Chiba et al., 1993, 1995). Although the molecular basis for cellular recognition is not well understood, it is thought to involve the differential expression of cell adhesion molecules by motoneurons and/or muscle fibers (Halpern et al., 1991; Nose et al., 1992; Chiba et al., 1995), as well as the avoidance of local repulsive signals (Nose et al., 1994; Matthes et al., 1995).

Although Drosophila neuromuscular connectivity is normally precise, at least two types plasticity have been observed at the neuromuscular junction: changes in connectivity and changes in ending morphology. Changes in connectivity can occur either in response to the loss of motoneuron innervation as in prospero or Toll mutants (Broadie and Bate, 1993b; Halfon et al., 1995) or through reduced synaptic activity (Jarecki and Keshishian, 1995). In addition, delays in normal innervation or reduced activity results in the appearance of collateral sprouts from adjacent nerves in the embryo (Jarecki et al., 1995; Kopczynski et al., 1996). Furthermore, morphological plasticity has been seen in hyperactive mutants that exhibit increased size and complexity of their larval motor endings arbors (Budnik et al., 1990; Zhong et al., 1992; Jia et al., 1993).

We have examined the peripheral innervation changes that occur after the ablation of individual motoneurons in the embryo using a microbeam laser. We focused on a well characterized motoneuron, RP3, and its target muscle fibers, 7 and 6 . The RP motoneurons have been described in detail with respect to their cell body locations, trajectories out of the CNS, timing of muscle fiber innervation, and motoneuron ending morphology (Patel et al., 1987; Halpern et al., 1991; Sink and Whitington, 1991a,b; Broadie et al., 1993a; Chiba et al., 1993).

We show that, despite the stereotypy of normal Drosophila peripheral connections, denervation of muscle fibers results in the formation of functional synaptic connections from neighboring 
sources onto the denervated muscle fibers. These collateral inputs are evident by the 1 st larval instar. Our results indicate that motoneurons that have undergone normal synaptogenesis can respond to changes in the degree of innervation of nearby muscles.

\section{MATERIALS AND METHODS}

The wild-type Canton S stock of Drosophila melanogaster was used throughout this study. Embryonic and larval dissections, immunocytochemistry, and imaging were performed as described previously by Johansen et al. (1989a), Halpern et al. (1991), and Chiba et al. (1993).

Laser ablation. Embryos were collected from $1 \mathrm{hr}$ egg lays and dechorionated and mounted under a coverslip in \#700 halocarbon oil. Embryos suitable for laser ablation (late stage 15 to early stage 16) were selected using the morphological criteria defined by Campos-Ortega and Hartenstein (1985). The laser ablation of RP motoneurons was performed using laser lesioning methods described by Cash et al. (1992). RP3 was imaged through the ventral surface of dechorionated embryos. The motoneuron was ablated using a Laser Sciences (Cambridge, MA) VSL 337 nitrogen laser, coupled to a dual-mirror dye cell module emitting at $440 \mathrm{~nm}$ with peak pulse energy of $27 \mu \mathrm{J}$ and peak power of $9 \mathrm{~kW}$. Laser light was focused with a $63 \times$ oil planapochromat objective to an $\sim 2 \mu \mathrm{m}$ spot, and intensities were adjusted using neutral density filters. Immediate signs of cell death were based on cell body blebbing at the time of laser ablation. The extent of local cell death after laser ablation of RP3 was gauged $1 \mathrm{hr}$ after the operation by vitally staining filleted embryos with $0.5 \%$ trypan blue in saline. Similarly, the specificity of the ablations for the RP motoneurons was determined in filleted embryos using Fasciclin III immunocytochemistry (Halpern et al., 1991). Operated embryos were recovered from the halocarbon oil, transferred to food plates, and subsequently dissected at various stages of development.

Electrophysiology. Glutamate iontophoresis and intracellular recording were performed as described by Cash et al. (1992), except that motor endings in larval fillets were vitally labeled fluorescently by incubating for $1 \mathrm{hr}$ at room temperature with FITC-labeled anti-HRP at 1:100 dilution (Cappel, West Chester, PA) in saline, followed by a 30 min wash in fresh saline. The body walls were briefly scanned fluorescently so that nerves and ectopic motor endings could be located. Both native and foreign nerves to the muscle fibers were independently excited by extracellular suction pipettes, with the intracellular response monitored in currentclamp mode. In addition, visualized boutons were individually excited with iontophoretically applied glutamate $\left(0.5 \mathrm{M}\right.$ glutamate in $\left.\mathrm{dH}_{2} 0\right)$ as described previously by Johansen et al. (1989a) and Cash et al. (1992).

Statistical analysis. All determinations of branch number, innervation type, muscle dimensions, and innervation extent were made with the aid of digitally enhanced video microscopy. To determine the approximate area covered by neuromuscular endings on a muscle, a $20 \times 5$ grid was superimposed on the monitor display to the dimensions of each fiber. Grid rectangles were approximately square. The number of rectangles (out of the total 100) into which each motor ending process crossed was counted to give a measure of extent. No rectangle was counted twice. One rectangle was added to the count if an ending exactly intersected a grid cross-hatch.

\section{RESULTS}

\section{Identification of motoneurons and ending nomenclature}

Electrophysiological (Jan and Jan, 1976; Broadie and Bate, 1993a; Kurdyak et al., 1994) and anatomical (Atwood et al., 1993; Kurdyak et al., 1994) studies indicate that muscle fibers 7 and 6 are innervated by up to two motoneurons, of which one is invariably the motoneuron RP3 (Halpern et al., 1991; Sink and Whitington, 1991a,b; Broadie and Bate, 1993a; Chiba et al., 1993) (see Fig. $1 A, B)$.

These two inputs can be recognized by the morphological features of the motor endings on muscle fibers 7 and 6 in 3rd instar larvae. There are several lines of evidence that individual motoneurons have specific ending morphologies. In Drosophila, every muscle fiber is reliably innervated by its own complement of motoneurons that elaborate characteristic ending morphologies
(Johansen et al., 1989a,b; Halpern et al., 1991). In the larva, these neuromuscular endings fall into at least three categories, type Ib and type Is, which have large and intermediate sized boutons, respectively, and type II, which have smaller boutons and are generally more branched and expansive. In addition, backfills from individual boutons (Cash et al., 1992) and neuron-specific immunocytochemistry (Monastiriati et al., 1995) show that a given neuron has only one ending type even when it innervates multiple targets. Finally, motoneurons deprived of their native target fibers not only synapse with neighboring fibers but also maintain their native ending type (Cash et al., 1992), suggesting that this phenotype is intrinsic to the neuron.

In 3rd instar larvae, there are two anatomically distinct motoneuron arbors on muscle fibers 7 and 6, termed ending types $\mathrm{Ib}$ and Is by Kurdyak et al. (1994) (Fig. 1). Both arbors arise at the cleft between the two muscle fibers. We observed that the type Ib motoneuron arbor is always present in each abdominal segment from A2 to A7 on both muscle fibers 7 and $6(n=89$ hemisegments, 8 animals), consistent with the innervation profile of RP3 (Halpern et al., 1991). The other, type Is motoneuron arbor is usually present in anterior segments, but is more variable in posterior segments (100\% frequency in A2-A3 compared to $50 \%$ in segment A7). Based on dye-fills and electrophysiological evidence, the type Is motoneuron located on muscle fibers 7 and 6 projects to several other ventral longitudinal muscle fibers, including muscle fibers 12 and 13 (G. Lnenicka and H. Keshishian, unpublished data) (Fig. 1A; gray axon). These observations suggest that the type Ib motoneuron arbor on muscle fibers 7 and 6 corresponds to motoneuron RP3, whereas the more variable Is arbor corresponds to the second motoneuron. A similar suggestion has been made by Kurdyak et al. (1994).

\section{Ablation of motoneurons innervating muscle fibers 7 and 6}

To test the effect of motoneuron denervation on synaptic connectivity, we laser-ablated RP motoneurons in dechorionated late stage 15 and early stage 16 embryos. At this stage of development, the RP3 motoneuron growth cone has just initiated contact with its two muscle fiber targets, 7 and 6 (Halpern et al., 1991; Sink and Whitington, 1991a,b; Broadie and Bate, 1993a). The operated embryos were recovered and allowed to develop up to the 3rd instar. They were then labeled using neuron-specific immunocytochemistry (anti-HRP) to examine the effects of denervation on neuromuscular connectivity and development.

Using digital enhancement techniques (Halpern et al., 1991), it is possible to image the RP3 cell bodies within the CNS of live embryos (Fig. 2A). The RP3 cell bodies (arrowhead) can be recognized by their characteristic location between the anterior and posterior commissures, medial to the longitudinal connectives (Fig. 2A, $a c, p c, l c$ ). By focusing dorsally within the CNS, it is also possible to image the cell bodies of RP1 and RP4, the two motoneurons that lie adjacent to RP3 and that project to muscle fiber 13 (Fig. $1 A$; see also Fig. $2 C$ ). In Figure $2 A$, the small rectangle indicates the approximate target size of the laser microbeam $(1 \times 2 \mu \mathrm{m})$, superimposed on the video image of a live stage 15 embryo. Using this method, we targeted every visible RP3 motoneuron in abdominal segments on one side of the CNS, leaving the other side as an unoperated control. The operation should at least partially denervate muscle fibers 7 and 6 in abdominal segments on the side contralateral to the ablated RP cell bodies.

We tested the accuracy and specificity of the laser ablation by 

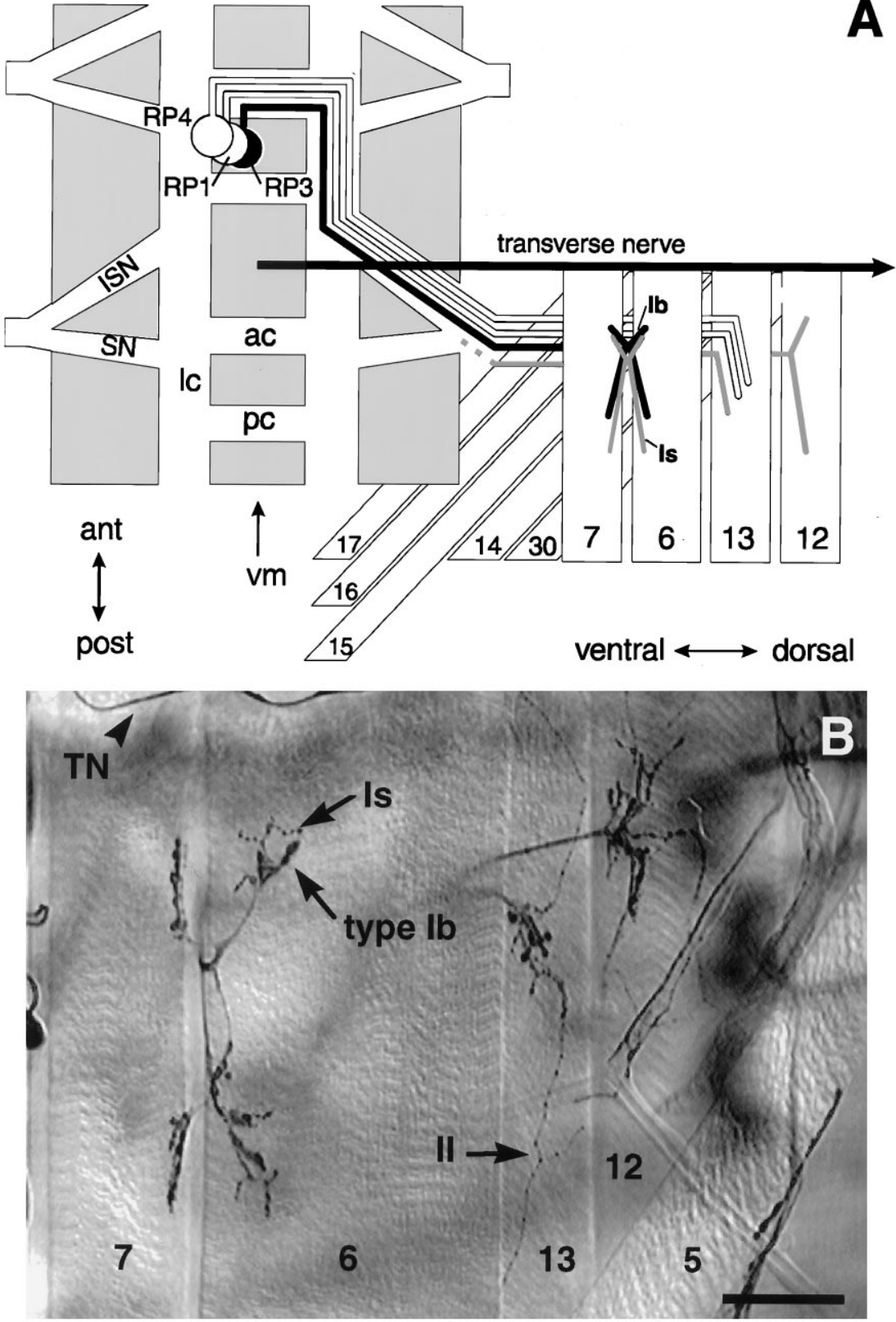

Figure 1. The cell body locations, muscle targets, and axonal pathways of RP motoneurons discussed in this study. $A$, Schematic drawing of one abdominal hemisegment showing the pathway for $R P 3$, and the neighboring motoneurons $R P 1$ and $R P 4$. The segmentally repeated RP3 motoneuron cell bodies are located on the dorsal aspect of the CNS (gray) on either side of the midline ( $\mathrm{vm}$ ). Each RP3 axon (black) crosses the midline in the anterior commissure $(a c)$ and turns posteriorly in the longitudinal connective $(l c)$ to exit the CNS via the intersegmental nerve tract $(I S N)$. There it joins the segmental nerve $(S N)$ to innervate muscle fibers 7 and 6 in the next posterior segment. The axon projects within the cleft between the two fibers to establish a characteristic nerve branch and site of innervation at stage 16 (Halpern et al., 1991; Sink and Whitington, 1991a,b; Broadie and Bate, 1993a) (for review, see Broadie et al., 1994). The second input to the $7 / 6$ cleft (gray) also innervates other ventral muscle targets. Its cell body location is not established. RP1 and RP4 continue laterally to innervate muscle fiber 13 (Halpern et al., 1991; Sink and Whitington, 1991a). The transverse nerve $(T N)$ runs along the borders of each segment. $B$, The ventral musculature of a 3rd instar larval fillet stained for motoneurons with an antibody to horseradish peroxidase. There are at least three morphologically distinct ending types: type $I b$ (large boutons), type $I s$ (intermediate-sized boutons), and type II (small boutons, more branched and extensive). These have been shown by bouton backfills to belong to distinct motoneurons. Note that Drosophila muscle fibers can be polyinnervated. Scale bar, $50 \mu \mathrm{m}$. labeling the RP motoneurons and scoring their numbers after the operation. In embryos the motoneurons RP1, RP3, and RP4 express the cell adhesion molecule Fasciclin III on their cell body surfaces (Patel et al., 1989; Snow et al., 1989; Halpern et al., 1991; Chiba et al., 1993). Using immunolabeling, the individual RP motoneurons were identified and counted in embryonic fillet preparations (Halpern et al., 1991; Halfon et al., 1995). Figure 2, $B$ and $C$, shows a single segment of the CNS at two focal levels after an RP3 ablation. In Figure $2 B$, the white arrow points to a gap on the operated side where the RP3 cell body would normally be located. On the contralateral side, an intact, RP3 cell body stained for FasIII is visible (black arrowhead). In Figure $2 C$, the same segment is shown focused dorsally at the level of motoneurons RP1 and RP4, which appear undamaged on both the operated and the control sides. In a sample study of 10 embryos, we found that $91 \%$ of the hemisegments on the operated side had missing RP3 motoneuron cell bodies, compared to $4 \%$ on the control side of the same animals. However, we also found that many of the operated hemisegments had missing RP1 and RP4 motoneurons. Thus, whereas in $44 \%$ of the hemisegments only RP3 was ablated, in another $37 \%$ of the hemisegments both RP3 and RP4 were absent, and in another $11 \%$ all three RP motoneurons were missing ( $n=46 \mathrm{CNS}$ segments). To check the gross extent of laser damage, we examined embryos dissected within 1 hr after ablation using the vital dye trypan blue, which stains necrotic cells. We observed in the CNS, in each operated hemisegment between 1 to 3 blue, necrotic cells, consistent with the Fasciclin III results ( $n=20$ segments examined; data not shown).

\section{Laser ablation of the RP motoneurons results in partial} or complete cleft denervation of muscle fibers 7 and 6

Using the ablation methods described above, we examined the effects of embryonic motoneuron ablation in 1st instar larvae and in mature 3rd instar larvae. Laser ablation directed at RP3 resulted in the partial or complete loss of innervation at the normal cleft input site on muscle fibers 7 and 6 , which was evident in both 

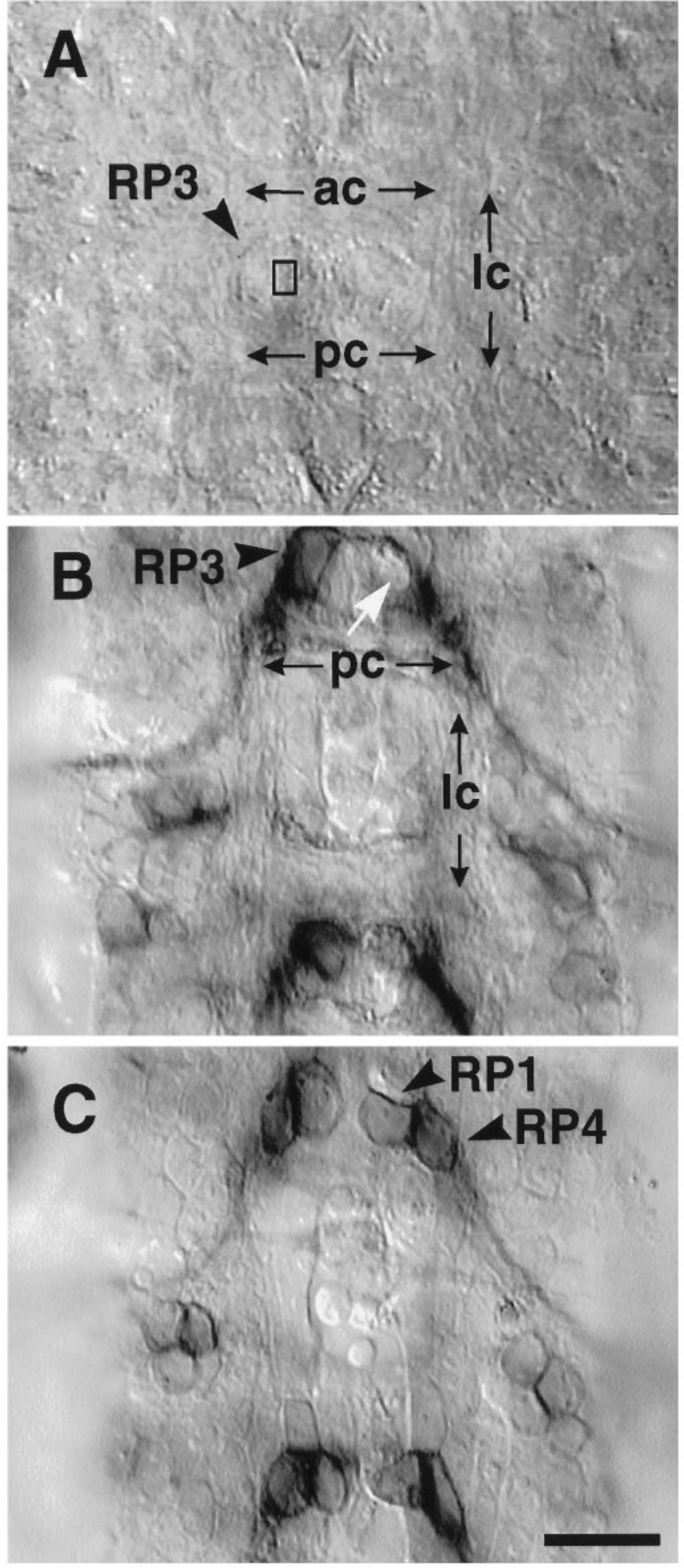

Figure 2. Laser ablation of RP3 in live stage late $15 /$ early 16 embryos. $A$, The cell body of RP3 is imaged through the ventral surface of the dechorionated embryo. RP3's cell body is located near the dorsal (internal) surface of the ventral nerve cord. Two RP3 cell bodies (arrowhead) can clearly be seen flanking the midline and bordered by the lateral commissures ( $l c)$ on the left and right, and above and below by the anterior and posterior commissures $(a c, p c)$. The rectangle indicates the $\sim 1 \times 2 \mu \mathrm{m}$ target site of the laser. Every visible RP3 on one side was targeted. The contralateral RP3 seconds were left intact as internal controls. $B, C$, To test the specificity of the laser ablation with respect to neighboring cells, operated embryos were acutely filleted and stained with anti-Fasciclin III, which labels a subset of neurons, including $R P 1, R P 3$, $R P 4$. B, In this segment seen from the dorsal view, there is no staining in the location of RP3 (white arrow), whereas the contralateral RP3 is still immunopositive for FasIII. $C$, At the dorsal surface of the CNS, one cell layer above RP3, both $R P 1$ and RP4 can be seen on both sides. Scale bar, $10 \mu \mathrm{m}$. 1st and 3rd instar larvae. We defined a complete denervation as cases in which the normal innervation at the cleft between muscle fibers 7 and 6 is absent (see Fig. $1 B$ for the normal pattern). Partial denervation was defined by the absence of either of the two (type Ib or Is) motoneuron arbors normally present in the cleft. Because the Is motoneuron arbor is variably present in posterior segments of control animals, we confined most of our analysis of denervation to segments $\mathrm{A} 2$ and $\mathrm{A} 3$, where the innervation is not variant. In the 1st instar larvae, only complete denervation was analyzed because the relatively undifferentiated appearance of the synaptic boutons did not allow us to distinguish two distinct ending types.

Figures 3, $A$ and $B$, shows control and experimental abdominal segments of the same 1 st instar embryo. In Figure $3 A$, the black arrow points to the normal nerve entry point and arborization in the cleft region, with motoneuron branches contacting both muscle fibers 7 and 6 . By contrast, on the contralateral side of this segment (Fig. 3B) there is no SNb branch at the cleft and the two fibers have separated slightly (arrowheads). In laser-treated animals examined in the 1st instar, we saw $25 \%$ complete denervation of muscle fibers 7 and 6 on the operated side, compared to $0 \%$ on the control side $(n=16$ larvae, 60 hemisegments, segments A2-A3).

We performed most of our analysis in 3rd instar larvae where two distinct bouton types can be readily distinguished. Muscle fibers that have no native innervation are easily identified by the complete absence of a motoneuron arbor at the 7/6 cleft (Fig. $3 B, D)$. We also observed 3rd instar hemisegments with apparent partial native denervation, evidenced by the absence of either the $\mathrm{Ib}$ or the Is motoneuron arbor. In segments A2-A3, where both inputs are normally present in control animals, we found that on the experimental side $51 \%$ of the muscle fibers 7 and 6 were missing their cleft Is or Ib motoneuron arbors after RP3 operations, with another $30 \%$ showing complete cleft denervation in these two segments, and the remaining $19 \%$ having both arbors. This $81 \%$ frequency of partial or complete denervation on the operated side compared to $14 \%$ on the control side (Fig. $4 A$ ).

There are normally two motoneuron inputs to muscle fibers 7 and 6 in abdominal segments 2 and 3 (Fig. 1). Nevertheless, in $30 \%$ of the hemisegments an ablation directed at one of these neurons (RP3) resulted in the absence of both inputs. This suggests that the ablation may also damage the second cell. However, it has been reported that the cell body and axon of the second motoneuron are located ipsilaterally in the CNS (Keshishian et al., 1993; Chu-LaGraff et al., 1995) and, therefore, would not be near the laser target. Alternatively, because the second axon arrives at the target several hours after RP3 (Broadie and Bate, 1993a), it is possible that it depends on RP3 to pioneer its projection to the target site. Further analysis of the development of the second motoneuron will help resolve this issue.

Because the Ib input at the $7 / 6$ cleft is present in all abdominal segments in control animals, its loss from the cleft may reflect the success of RP3 ablation. We found that the Ib input was missing at the $7 / 6$ cleft in $48 \%$ of hemisegments on the experimental side compared to $8.6 \%$ on the control side $(n=213$ rd instar larvae, 194 hemisegments, segments A2-A7). However, our results showed a higher success rate in ablating RP3 when assessed in the embryo shortly after the operation $(90 \%)$. This discrepancy could be attributable to an underestimate of the degree of denervation in the larvae, caused by the presence of ectopic inputs located in the cleft that are indistinguishable from native inputs (see below). 

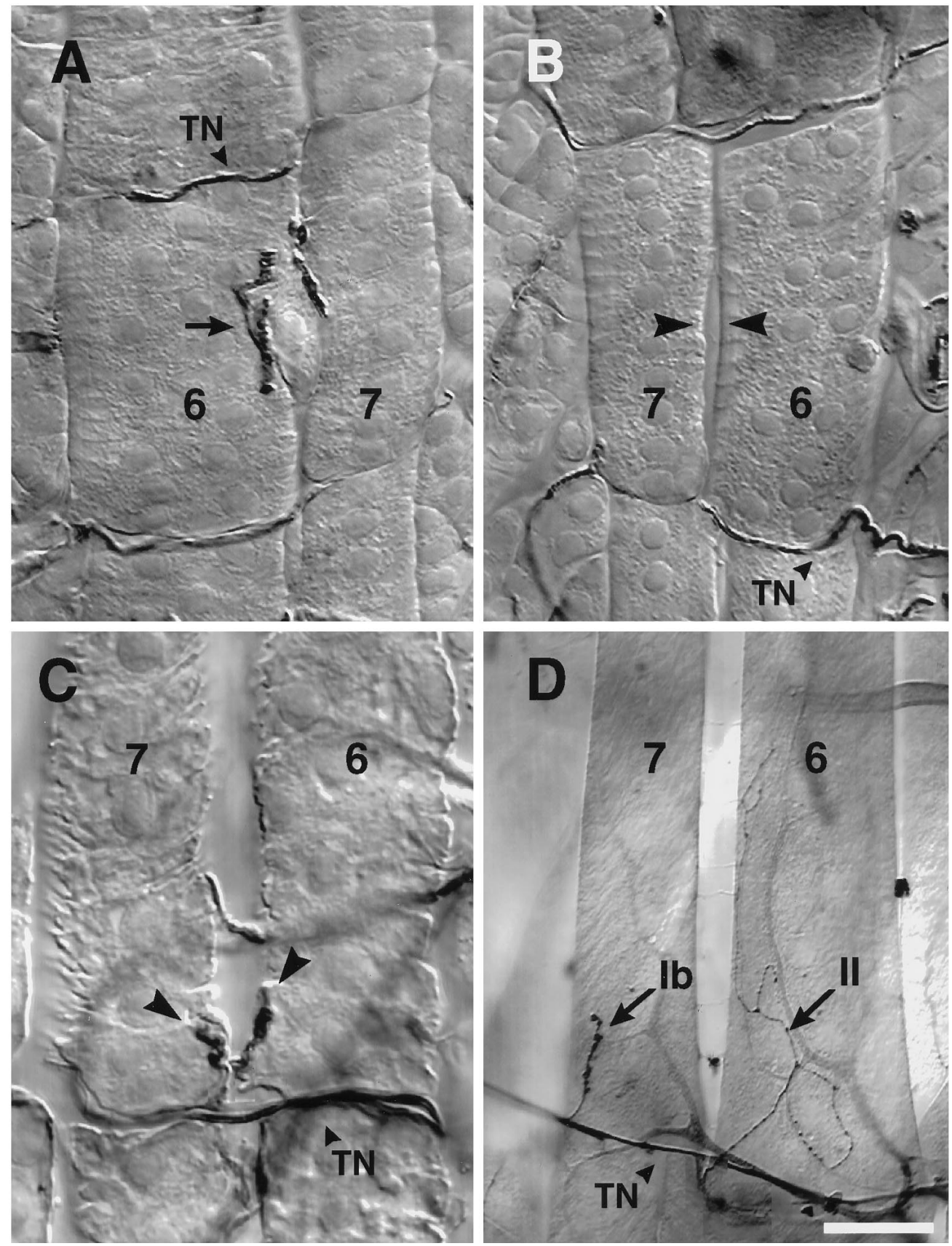

Figure 3. Ablation of RP3 leads to denervation of target muscle fibers 7 and 6 and the appearance of ectopic inputs. $A-C$, 1st instar larvae, $(D)$ 3rd instar. $A$ and $B$ show, respectively, the left and right ventral hemisegment of a 1st instar larva after embryonic laser ablation. In $B$, the cleft is denervated on the side where RP3 normally innervates (arrowheads). On the contralateral untreated side of the same animal $(A)$, the $7 / 6$ cleft is innervated with well defined boutons characteristic of 1st instar control animals. $C$, Collateral inputs (arrowheads) from the transverse nerve onto the denervated 7 and 6 muscle fibers of a 1st instar larva. $D$, Ectopic inputs on a cleft-denervated 3 rd instar hemisegment. Indicated are a type $I b$ input from the transverse nerve onto the posterior end of muscle fiber 7 , and a type $I I$ input onto muscle fiber 6 , traced back to the input on the next posterior muscle fibers 15/16 (source not shown). Scale bars: $A-C, 10 \mu \mathrm{m}$; $D, 40 \mu \mathrm{m}$

\section{Denervation is associated with the appearance of ectopically located foreign synapses}

An important consequence of the RP kills was the appearance of collateral branches and novel motor endings on the denervated muscle fibers. In untreated control 3rd instar larvae, ectopically placed inputs are rare $(5.6 \%$ of hemisegments in 8 larvae, 89 hemisegments). In laser-treated animals examined in the 1st instar, $80 \%$ of completely cleft-denervated hemisegments had ectopically placed inputs ( $n=15$ of $86 \mathrm{~A} 2-\mathrm{A} 7$ hemisegments, 16 1st instar larvae) compared to $4.7 \%$ of hemisegments with endings on both 7 and 6 . Similarly, in 3rd instar larvae, 93\% of hemisegments that were completely $7 / 6$ cleft-denervated had ectopic inputs ( $n=$ 42 of 198 A2-A7 hemisegments, 21 larvae). This frequency of ectopic innervation was not significantly different from that seen in 1st instar experimental larvae.
Figure 3C (arrowheads) illustrates the small, ectopically placed contacts visible in 1st instar larvae after denervation in the embryo. The ectopic contacts emerge from the transverse nerve, a mixed motor/sensory projection that runs from the dorsal midline of the CNS along the segment borders, which includes motoneurons projecting to muscle fibers 25 (Gorczyca et al., 1994) and 8 (Cantera and Nassel, 1992). In Figure 3D, a denervated 3rd instar larva is shown with a collateral type Ib projection from the transverse nerve, which has innervated muscle fiber 7. Also shown is a type II projection onto muscle fiber 6 from the ending at the next posterior muscle fiber 15 and 16 cleft.

Complete denervation was correlated with increased numbers of ectopic inputs. Figure $4 B$ illustrates the frequency of ectopic contacts on muscle fibers 7 and 6 in segments A2-A3 in 3rd instar larvae, as a function of the degree of denervation. For segments in 




Normal Cleft Innervation

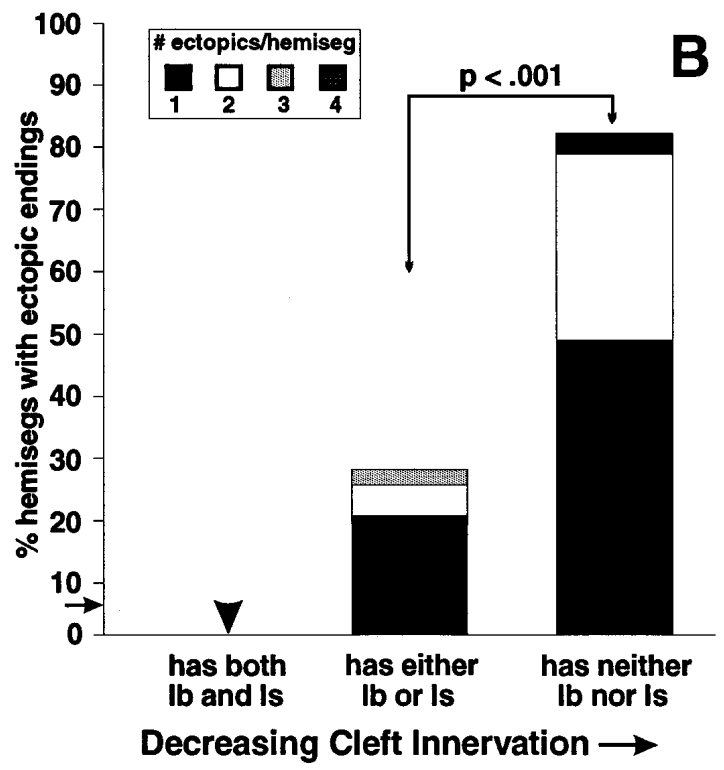

Figure 4. Reduction in innervation at muscle fibers 7 and 6 leads to the appearance of collateral inputs. $A$, In control animals, hemisegments A2-A3 always have both type Ib and type Is inputs at the cleft (frequency shown by arrow; $n=32$ hemisegments, 8 larvae). In contrast, in lasertreated animals, on the experimental side (contralateral to laser ablation) a significantly smaller number of hemisegments had both cleft inputs present at the $7 / 6$ cleft than on the control side (ipsilateral to laser ablation; $n=21$ larvae, 74 hemisegments, A2-A3). B, Increased cleft denervation correlates with an increased probability and frequency of collateral innervation. Muscle fibers 7 and 6 are examined in segments A2-A3. The black bars indicate the percent of hemisegments with ectopic endings that have only one ectopic ending. Bar heights indicate total percent of hemisegments with ectopic endings in each group. From left to right: hemisegments that have both Ib and Is at the cleft ("normal" cleft innervation; $n=6$ hemisegments), hemisegments that have one or the other (partial innervation; $n=41$ hemisegments), and hemisegments that have neither (complete denervation; $n=27$ hemisegments, 21 lasertreated larvae examined in A2-A3). In control animals, ectopically placed inputs were seen at low frequency (shown by arrow; $n=89$ hemisegments, 8 animals).

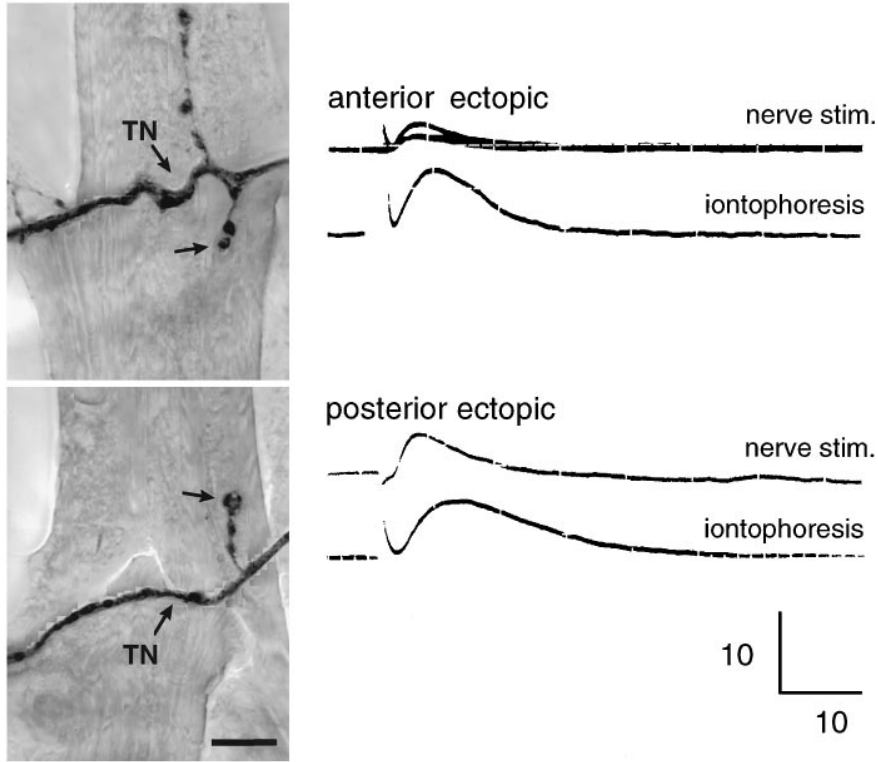

Figure 5. Ectopic inputs are physiologically functional. After embryonic laser ablation, 3rd instar larvae were filleted in insect saline and incubated with fluoresceinated anti-HRP to vitally image ectopic inputs on denervated muscle fibers 7 and 6 . The pictured ectopic inputs from the transverse nerve $(T N)$ are located at the anterior and posterior ends of the same muscle fiber 7. An intracellular electrode in the muscle fiber recorded a postsynaptic potential in response to both shocking the transverse nerve using a suction electrode (top trace) and iontophoresis of glutamate above the boutons (bottom trace; scale shown is $10 \mathrm{mV}, 10$ msec). The fillet was then fixed and relabeled with anti-HRP. The results indicate that both pre- and postsynaptic elements of these collateral inputs are functional. Scale bar, $10 \mu \mathrm{m}$.

which both muscle fibers 7 and 6 were innervated with two motoneuron arbors at the cleft, none had ectopic motor endings, compared to the $5.6 \%$ frequency observed in unoperated control larvae. However, when only a single motoneuron arbor was seen at the cleft, the fraction of the muscle fibers receiving ectopic contacts increased to $27 \%$, with the contacts averaging 1.5 per fiber. Finally, when the muscle fibers were completely denervated at the cleft, the frequency of ectopic contacts rose to $82 \%$ of the hemisegments, with an average of 1.4 foreign contacts per fiber. These data show that contrary to the situation in unoperated animals, where motoneurons retain their target specificity throughout larval life, and ectopically placed foreign contacts are rare, denervation leads to a local alteration in motoneuronal projections.

\section{Denervation induced collateral endings are functional}

To determine whether the novel, ectopically placed contacts were synaptically functional, we examined them electrophysiologically using both evoked nerve excitation and neurotransmitter iontophoresis (Fig. 5). After laser ablation of RP motoneurons in the embryo, the motor endings of the 3rd instar larva were examined vitally by incubating the larval fillets with fluorescently labeled anti-HRP. In this way, denervated muscle fibers bearing ectopic contacts were identified in live preparations before electrophysiological study. Figure 5 shows transverse nerve-evoked PSPs and local glutamatergic iontophoretic potentials recorded from two ectopic contacts located on either end of a denervated muscle fiber 6 . These results ( $n=3$ larval preparations) indicate that the foreign contacts made on denervated muscle fibers were physiologically functional and capable of evoking PSPs. Furthermore, 


\begin{tabular}{|c|c|c|c|c|c|c|}
\hline \multicolumn{7}{|c|}{$\begin{array}{l}\text { Table 1. Sources and types of ectopic inputs on muscle } \mathbf{f} \\
\text { and } 6(\%) \\
\qquad \text { Percent distribution of sources }\end{array}$} \\
\hline & $\mathrm{TN}$ & $15 / 16$ & Next $7 / 6$ & $14 / 30$ & $13 / 12$ & Other \\
\hline \multicolumn{7}{|l|}{ All types: Ib, Is, II } \\
\hline Muscle fiber 7 (65\%) & 58 & 25 & 6.3 & 1.8 & 0 & 9.0 \\
\hline Muscle fiber $6(35 \%)$ & 18 & 4.9 & 25 & 9.8 & 25 & 18 \\
\hline \multirow[t]{3}{*}{7 and $6(n=172)$} & 44 & 18 & 13 & 4.7 & 8.7 & 12 \\
\hline & \multicolumn{6}{|c|}{ Percent distribution by ending type } \\
\hline & $\mathrm{TN}$ & $15 / 16$ & Next $7 / 6$ & $14 / 30$ & $13 / 12$ & Other \\
\hline \multicolumn{7}{|l|}{ Type $1 b$} \\
\hline Muscle fiber $7(72 \%)$ & 73 & 17 & 0 & 0 & 0 & 10 \\
\hline Muscle fiber $6(28 \%)$ & 11 & 5.3 & 26 & 16 & 37 & 5.3 \\
\hline 7 and $6(n=67)$ & 55 & 13 & 7.5 & 4.5 & 10 & 9.0 \\
\hline \multicolumn{7}{|l|}{ Type Is } \\
\hline Muscle fiber 7 (75\%) & 85 & 11 & 3.7 & 0 & 0 & 0 \\
\hline Muscle fiber $6(25 \%)$ & 44 & 0 & 11 & 22 & 22 & 0 \\
\hline 7 and $6(n=36)$ & 75 & 8.3 & 5.6 & 5.6 & 5.6 & 0 \\
\hline \multicolumn{7}{|l|}{ Type II } \\
\hline Muscle fiber 7 (52\%) & 17 & 47 & 17 & 5.6 & 0 & 14 \\
\hline Muscle fiber $6(48 \%)$ & 15 & 6.1 & 27 & 3.0 & 18 & 30 \\
\hline 7 and $6(n=69)$ & 16 & 28 & 22 & 4.3 & 8.7 & 22 \\
\hline
\end{tabular}

TN, Collateral branch from the transverse nerve; 15/16, collateral branch from the ending on muscle fibers $15 / 16$, etc.; $n$, number of ectopic endings; 21 laser-treated larvae examined.

Each row indicates the distribution of sources for collateral endings as a percent of total collaterals seen. Data are also subdivided to indicate the different distributions on muscle fibers 7 vs 6 , as well as the distributions of collateral inputs of different ending type. The data indicate that frequencies for endings of different source and type vary widely depending on the target. For instance, muscle fiber 7 receives more collateral innervation than does muscle fiber 6 .

the muscle glutamate sensitivity was localized to the varicosities on the ectopic motor endings, indicating that they are functional boutons. We have also found that the ectopic motor endings have immunoreactivity to the neurotransmitter glutamate $(n=3$ hemisegments), the vesicle-associated protein synaptotagmin $(n=$ $3)$, and the cell adhesion molecule fasciclin II $(n=9)$, which is expressed by all motoneurons (data not shown). These observations indicate that the ectopic endings have immunological and physiological features associated with functional motoneuron endings.

\section{Frequency and distribution of ectopic endings}

The ectopic motor endings were derived from neighboring nerves or from motor endings located on adjacent muscle fibers (Table 1), but otherwise resembled normal Drosophila motor endings, with varicosities and branch morphologies similar to those seen in unoperated control larvae. The most common source $(44 \%)$ of the ectopic endings was the transverse nerve (Figs. $3 B-D, 6 B$ ), which runs on the internal surface of muscle fibers 7 and 6 along the boundary between abdominal hemisegments (Fig. 1A,B). The next most common source (18\%) of ectopic inputs was the motor ending from the ventral oblique muscle fibers 15 and 16 (Fig. $6 A, C)$. The remaining ectopic contacts were derived from other adjacent motor endings, including $13 \%$ that crossed segment borders from the next anterior or posterior muscle fibers 7 or 6 to reach the denervated fibers. As a rule, ectopic contacts arose from sources no greater than the width of one muscle fiber from the denervated fibers. The motor ending on the ventral oblique mus- cles 14 and 30 is situated directly underneath the 7/6 cleft. Collateral inputs from this terminal were probably sometimes miscounted as native cleft inputs because a separate branch from the $14 / 30$ ending could not always be distinguished. Consistent with the fact that our denervation protocol could additionally ablate RP1 and/or RP4, we also occasionally observed apparent partial denervation of muscle fiber 13 and the appearance of collateral inputs on this fiber (data not shown). This reduction in the normal innervation of muscle fiber 13 also could reduce the observed frequency of collateral innervation from muscle fiber 13 onto 6 .

\section{Evidence that the sprouting response is local}

To address whether laser ablation might cause a nonspecific sprouting response resulting in collateral innervation of normally innervated muscle fibers, we laser-ablated RP motoneuron cell bodies in every other segment of the embryonic CNS. We then examined neuromuscular innervation in the 3rd instar larvae and asked whether normally innervated segments adjacent to denervated segments received an increased number of collateral endings. We examined the response of the transverse nerve because it was equally in contact with both innervated and denervated muscle fibers, so that any effect attributable to differences in access and distance could be ruled out. In every case in which the transverse nerve formed a collateral input, the ending was made on the denervated hemisegment rather than the adjacent innervated hemisegment ( $n=15$ transverse nerve ectopics, 9 larvae, 92 experimental and control hemisegments). Collateral inputs from the transverse nerve were equally likely to innervate the anterior or posterior of the denervated muscle fibers.

In this experiment, $82 \%$ of hemisegments in which muscle fibers 7 and 6 were completely denervated at the cleft $(n=17$ of 92 experimental and control hemisegments in 9 larvae) had collateral innervation from any source, not significantly different from the 93\% frequency seen on cleft-denervated hemisegments in animals in which motoneurons were ablated in every hemisegment on one side. In $13 \%$ of hemisegments that were normally innervated $(n=$ 47 of 92 control hemisegments, 9 larvae), we saw innervation at ectopic sites, of which $6.4 \%$ arose from a collateral input on an adjacent, denervated hemisegment. This was not significantly different from the $5.6 \%$ frequency of ectopically placed endings seen in control animals. As in control animals, all ectopically placed endings on control hemisegments were type II, and all were small, with an expanse similar to that seen in control animals. Finally, the ectopic sources were the same in experimental animals laserablated in every other segment compared to those laser-ablated on one side.

\section{Type II cleft inputs increase in frequency and expanse}

In control unlasered animals, type II inputs on muscle fibers 7 and 6 are rare, appearing on $5.6 \%$ of hemisegments at the cleft and an additional $5.6 \%$ of hemisegments at other sites. In contrast, type I inputs are present at the cleft in $100 \%$ of hemisegments and were not found elsewhere on muscle fibers 7 or 6 . At both cleft and ectopic locations in control animals, the type II inputs are small, with an innervation expanse of $\sim 1 \%$ (see Materials and Methods). However, in laser-treated animals type II inputs to denervated fibers increased in frequency at both native and ectopic sites (Fig. 6D, Table 1). For example, type II inputs located at the cleft were observed at significantly higher frequency on hemisegments on the laser-treated side than on the contralateral control hemisegments in operated animals $(p<0.002 ; 22.3$ vs $12.6 \%$ in 21 larvae, 198 hemisegments) or when compared to 
Figure 6. Collateral inputs are formed on denervated muscle fibers from nearby motor axons and terminals. $A$, An example of collateral inputs from the muscle 15/16 cleft innervating muscle fiber 7 from the medial aspect. Both type $I b$ and type II endings are present. Arrowheads indicate the denervated cleft between muscle fibers 7 and 6 . The inputs visible between the arrowheads are on the underlying muscle fiber $14 . B$, The three different types of ending morphology are evident in this example: an Is ending from the transverse nerve $(T N)$ to the posterior end of the anterior muscle fiber 7 , a $\mathrm{Ib}$ input from $\mathrm{SNb}$ branching onto the lateral edge of muscle fiber 6 , and two type $I I$ inputs: one arising laterally from $\mathrm{SNb}$ onto the anterior muscle fiber 6 (source not shown), and the other laterally from $\mathrm{SNb}$ onto muscle fiber 6 (arrowhead). $C$, A type II input from the next posterior 15/16 cleft (arrowhead). Also visible are type Ib boutons that appear to be from the same source (see Fig. 7A). $D$, An example of a large, branched type II input from the $7 / 6$ cleft ramifying over both muscle fibers 7 and 6 (arrowheads). In contrast, type II inputs at the cleft in control animals are rare and small (see Results). Scale bar: $A, C, D, 30 \mu \mathrm{m}$; $B, 50 \mu \mathrm{m}$.
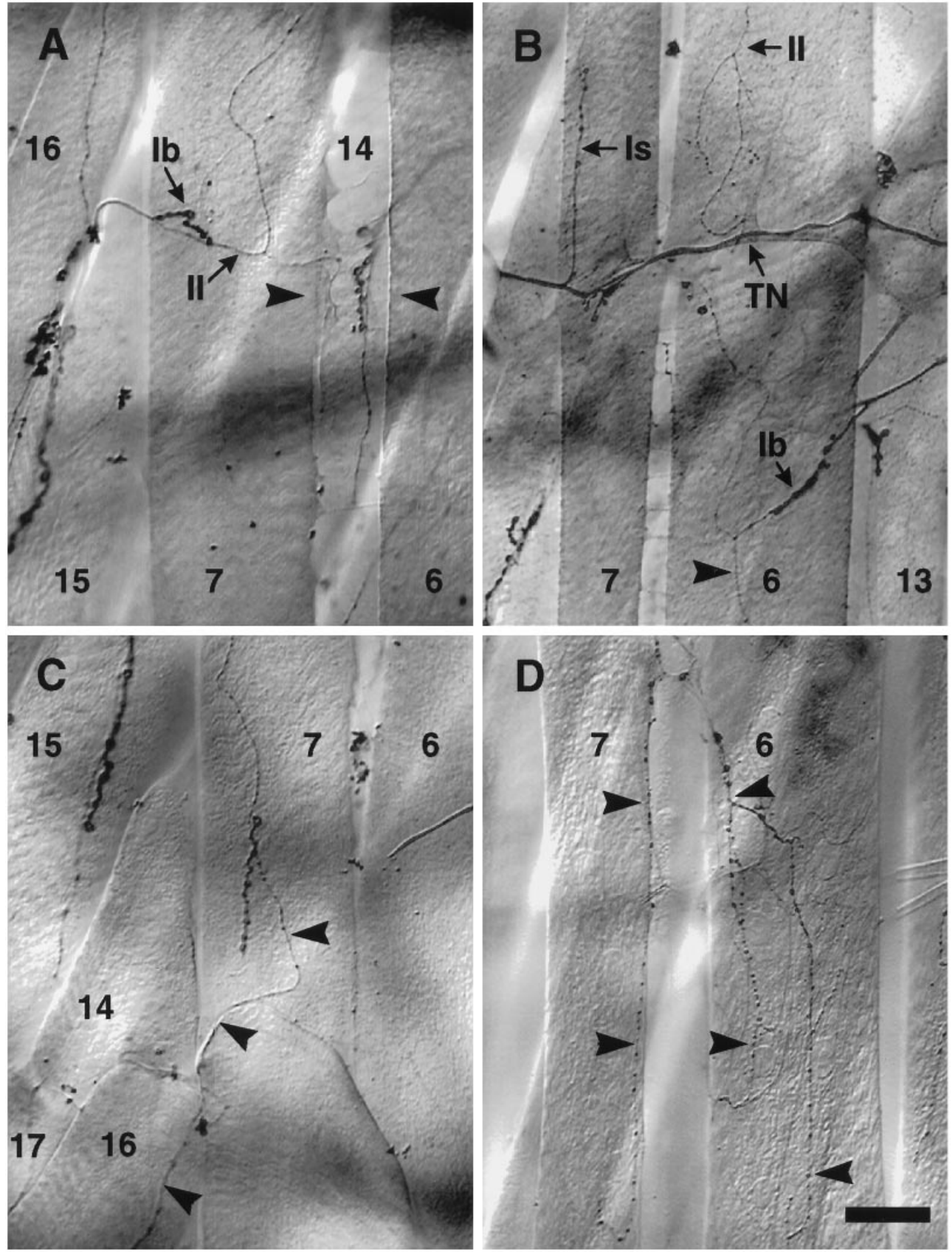

control unoperated animals $(p<0.001 ; 5.6 \%$ in 8 control larvae, 89 hemisegments). The frequency of hemisegments with type II cleft inputs on the control side was not significantly different from that found on the control animals. As in control animals, a few small cleft type II inputs were also seen on both sides. However, the experimentally induced cleft type II endings have an average expanse that is significantly greater than that seen in control animals (e.g., percent of muscle fiber area on experimental side muscle fiber 6 was $24.5 \pm 2.4$ vs $2.8 \pm 3.0$ in control animals). The experimentally induced expanse of cleft type II inputs is comparable to that normally seen on some muscles in control larvae (e.g., on muscle fiber 13 in control larvae, percent of muscle fiber area is $22.7 \pm 0.9$ ). Interestingly, the increase in type II expanse was seen both on the experimental side and on the few examples seen on the control side (e.g., $22.4 \pm 4.1$ on control side muscle fiber 6). This raises the possibility that there may also be a nonlocal response to denervation. However, the control side numbers were too few to test whether these more expansive cleft type II inputs were correlated with denervation.

Finally, as has been described in vertebrates (Tower, 1939) (for review, see Vrbova et al., 1995), denervation in Drosophila had the effect of reducing muscle fiber size. The width of muscle fibers on the experimental side was significantly reduced ( $p<0.001)$ compared to the contralateral control fibers in a segment specific fashion. The average muscle fiber 7 width decreased by $17 \%$ $(59.4 \pm 1.9$ vs $49.5 \pm 1.6 \mu \mathrm{m})$, whereas muscle fiber 6 width decreased by $14 \%(93.0 \pm 3.5$ vs $80.0 \pm 3.0 \mu \mathrm{m} ; n=19$ larvae, 80 hemisegments).

\section{DISCUSSION}

We have laser-ablated RP motoneurons on one side of the embryonic CNS, leaving the contralateral motoneurons as internal 
controls, to characterize the effect on target innervation. As in vertebrates, denervation in Drosophila induces collateral innervation. These foreign motor endings (1) arise from neighboring motor endings and nerves, (2) are present by the 1st larval instar, (3) are physiologically functional, and (4) are seen at higher frequencies on completely versus partially denervated muscle fibers. In addition, muscle fibers with reduced native innervation are smaller than their normally innervated contralateral fibers. Our results show that motor endings respond to changes in the degree of innervation of neighboring muscle fibers and can establish functional collateral inputs, probably in response to a cue from the target environment.

\section{Evidence that the signal for collateral sprouting acts locally}

Although collateral inputs can arise from a variety of motor endings and nerves, the sources are in general located within filopodial reach or axonal contact of the denervated fibers during late embryogenesis, when normal synaptic connections are established (Halpern et al., 1991; Sink and Whitington, 1991a,b; Keshishian et al., 1993). This suggests that a muscle-derived or locally diffusible signal might be a sufficient cue for collateral sprouting. However, proximity alone is seemingly insufficient to account fully for the pattern of ectopic synapses seen. For example, collateral endings from the transverse nerve are made onto muscle fiber 7 six times more frequently than those onto muscle fiber 6 , despite the fact that both fibers are in equal contact with the nerve and muscle fiber 7 is a thinner fiber with fewer nuclei. However, this preference might be explained by the fact that one of the motoneurons in the transverse nerve normally branches off the nerve medial to muscle fiber 7 , to innervate the superficial ventral muscle fiber 25 (Gorczyca et al., 1994), and so may have greater access to muscle fiber 7 than 6 .

Further evidence for the local nature of the sprouting response was obtained by ablating RP motoneurons in every other segment. This created a situation in which the muscle fibers on one side of a segment border were denervated, whereas those on the other side remained normally innervated. We used the transverse nerve as a gauge for whether denervation in one segment would cause sprouting onto normally innervated muscle fibers in an adjacent segment. The transverse nerve was examined because it is in equal contact with muscle fibers 7 and 6 in adjacent hemisegments, eliminating possible differences caused by access (Fig. 1). In every case, ectopic endings arising from the transverse nerve were made onto the denervated rather than the adjacent innervated muscle fibers, indicating that denervated fibers are preferred targets for the sprouting response. We also examined collateral inputs from all sources onto both innervated and denervated hemisegments and saw no significant increase in frequency or size of collateral innervation onto innervated segments over those in control animals. In addition, one-half of the collateral endings onto innervated muscles arose from collateral endings on denervated muscle fibers in adjacent hemisegments. This suggests that if there is any effect of the sprouting stimulus that is not specific to the denervated muscle fibers, it nonetheless acts in their immediate vicinity.

\section{Two forms of plasticity: changes in connectivity and morphology}

Type II motor endings show two distinct types of plasticity, responding to denervation by altering both their connectivity and their morphology. We analyzed type II collateral inputs as two separate classes: those located at the native cleft innervation site and those found at ectopic locations. In unoperated animals, type II innervation anywhere on muscle fibers 7 or 6 occurs at low frequency and is small. However, in the experimental group, type II inputs were more numerous and expansive than in the control animals at both cleft and ectopic locations.

Type II inputs in Drosophila are distinct in a number of ways. In addition to being glutamatergic (Johansen et al., 1989a), they are octopaminergic and are probably derived from only two motoneurons per hemisegment that innervate multiple muscle fibers in the body wall (Monastiriati et al., 1995). In invertebrates, octopaminergic neurons have been shown to modulate postsynaptic response to glutamate by stimulating adenylyl cyclase activity (Evans and O'Shea, 1977) (for review, see Evans, 1992). In addition, both hyperexcitable mutants and a mutation in dunce, which increases the amount of cAMP, show a preferential expansion of type II endings, resulting in increased branch number and length (Budnick et al., 1990; Zhong et al., 1992). Therefore, in our experiments denervation may be inducing a compensatory response in these neuromodulatory neurons.

Interestingly, whereas increased activity and cAMP result in terminal sprouting of type II, reduction of $\mathrm{Ca}^{2+} /$ calmodulindependent protein kinase II (CaM kinase II) results in terminal sprouting of type I endings (Wang et al., 1994), suggesting that different second-messenger pathways mediate distinct activitydependent plastic changes in nerve terminals. None of these studies reported a concomitant increase in collateral innervation. In addition, previous reports of collateral sprouting in Drosophila motoneurons (Halfon et al., 1994; Jarecki and Keshishian, 1995) did not report an increase in terminal branching, suggesting that different mechanisms may be involved (Fig. 7). A similar distinction may be present in vertebrates. For instance, insulin-like growth factor 2 (IGF-2) preferentially induces sprouting from nodes of Ranvier (Caroni and Grandes, 1990), whereas ciliary neurotrophic factor (CNTF) causes sprouting mainly at motor endplates (Gurney et al., 1992).

\section{Mechanisms of denervation-induced collateral sprouting}

In principal, either the downregulation of a repulsive signal on denervation or the upregulation or increased access to an attractive signal may lead to collateral sprouting. Both attractive (Kennedy et al., 1994) and repulsive (Luo et al., 1993; Puschel et al., 1995) cues have been demonstrated in vertebrate axonal outgrowth. In Drosophila, both cell adhesion and repulsion are also implicated in normal synaptogenesis. Our results indicate that the signal must be nonspecific to some degree, because all nearby axons and terminals were observed to sprout onto denervated muscles.

There is considerable evidence in vertebrate systems that denervated muscle provides a retrograde signal that results in collateral sprouting (Henderson et al., 1983) (for review, see Brown et al., 1981; Brown and Lunn, 1988; Vrbova et al., 1995). The signal for sprouting appears to be activity-dependent. For example, blocking nerve conduction with tetrodotoxin (Brown and Ironton, 1977; Dahm and Landmesser, 1988) or acetylcholine release or receptors by either botulinum toxin or $\alpha$-bungarotoxin (Watson, 1969, 1970; Holland and Brown, 1980) induces sprouting. Furthermore, directly stimulating denervated muscles suppresses sprouting (Brown and Holland, 1979). Factors that have been implicated in collateral sprouting include GAP-43, which is upregulated in the neuron during outgrowth and plasticity (Aigner et al., 1995), target-derived trophic factors such as insulin-like 

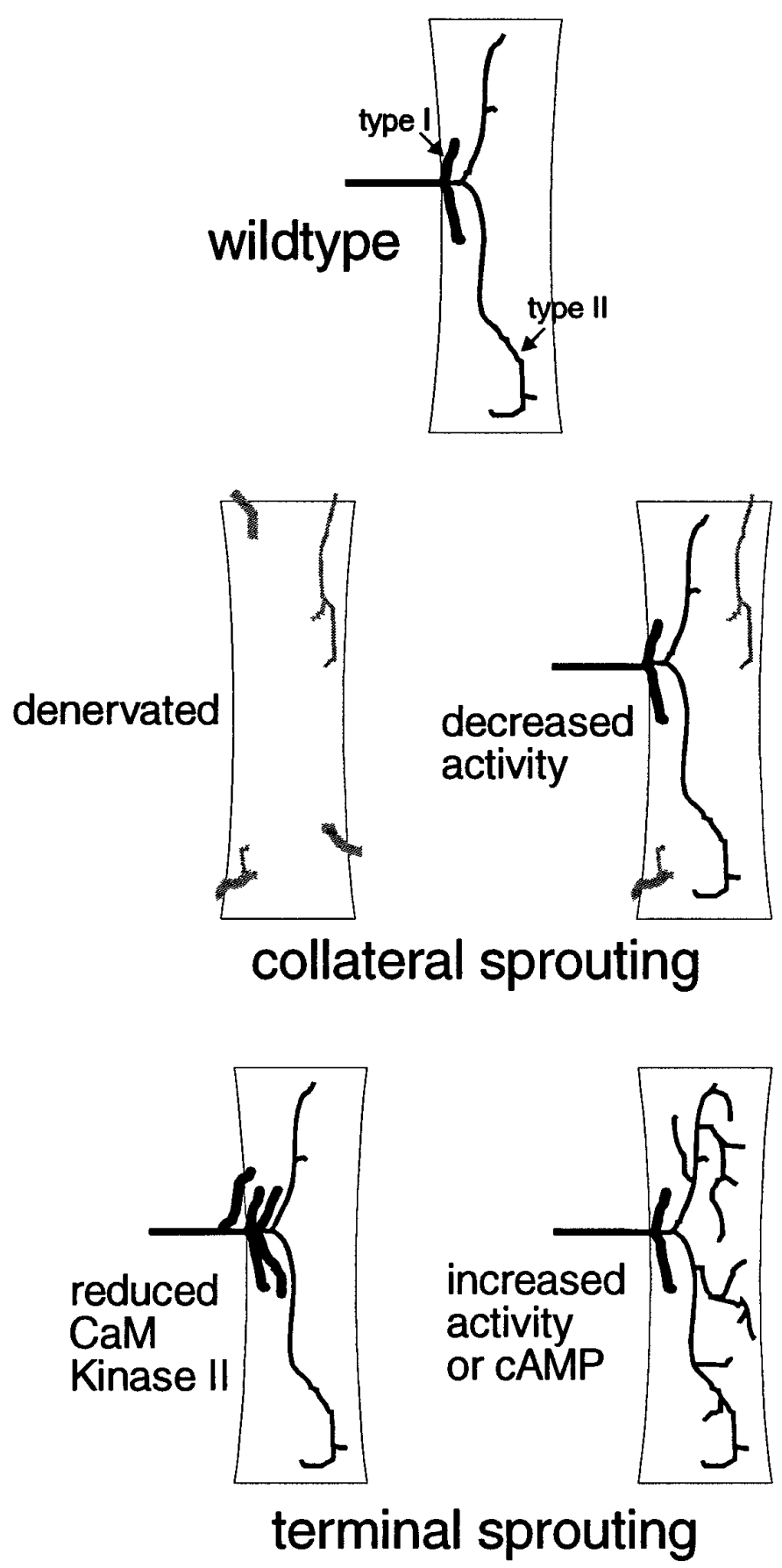

Figure 7. Summary of motoneuronal plasticity in Drosophila. Wild-type muscle fibers are innervated by type I (Ib and/or Is) motoneuronal endings. Additionally, they may have type II endings, which are more branched with smaller boutons. Collateral sprouting can result from either denervation (Halfon et al., 1994) (this study) or decreased activity (Jarecki and Keshishian, 1995). Terminal sprouting of type I endings can be caused by reduction of CaM kinase II, whereas terminal sprouting of type II endings can result from increased activity or increased cAMP (Budnik et al., 1990; Zhong et al., 1992).

growth factors (Caroni et al., 1994), and neurotrophic factors (Gurney et al., 1992; Shnell et al., 1994; Funakoshi et al., 1995).

Although no neurotrophic substance has yet been confirmed in Drosophila, activity has been shown to affect Drosophila neuromuscular development. For instance, there is good evidence that reducing presynaptic but not postsynaptic activity induces collat- eral innervation onto muscles that otherwise retain their normal complement of motoneuron inputs (Jarecki and Keshishian, 1995). The collateral endings described in that study are morphologically similar to those seen in this study and arise from the same array of sources. However, there are a number of differences. First, our experiment removes contact with the presynaptic ending. Second, the mutational analysis did not result in complete activity block, which may account for the smaller frequency of collateral inputs seen in that study. Finally, our experiment produced local denervation and local changes in connectivity allowing for internal controls, whereas the mutant analysis produced global changes in activity as well as connectivity. Nevertheless, the similarity in the results suggests that our data may be explained in part or entirely by the loss of presynaptic activity.

\section{Role of plasticity in normal development}

In the development of many vertebrate systems, initially coarse connections are refined by activity. Examples include neuromuscular synaptogenesis (Benoit and Changeux, 1975) (for review, see Thompson, 1986), formation of the visuotopic maps (for review, see Constantine-Paton et al., 1990; Shatz, 1990), and formation of cortical ocular dominance and orientation columns (Hubel et al., 1977) (for review, see Shatz, 1990). Although in Drosophila muscle targeting is precise, it occurs after a period of filopodial process withdrawal from inappropriate muscles (Halpern et al., 1991; Sink et al., 1991a,b), a form of morphological refinement whose importance for establishing final connections remains to be tested.

Our results show that the Drosophila neuromuscular system is capable of denervation-induced collateral innervation. It is possible that, similar to the situation in vertebrate systems, plasticity in normal Drosophila development ensures the fidelity of connections initiated by target recognition. Denervation may interfere with connectivity refinement, allowing nonspecific connections to be made or maintained. Therefore, our experiments may be unmasking an underlying process of refinement that normally occurs during late embryonic synaptogenesis. Drosophila has emerged as a preeminent system for studying learning and memory (Yin et al., 1994; Yin et al., 1995; Zhong, 1995). However, much less is known in this system about the structural changes associated with synaptic plasticity. Our results demonstrate forms of plasticity that may also be involved in other aspects of the Drosophila nervous system.

Our study raises a number of questions about the nature of plasticity in Drosophila. For instance, (1) we have demonstrated that denervation can induce both collateral and terminal sprouting. Other studies have shown that collateral innervation (Halfon et al., 1994; Jarecki and Keshishian, 1995) and terminal sprouting (Budnik et al., 1990; Zhong et al., 1992; Wang et al., 1994) can be induced by different stimuli (Fig. 7). Does partial denervation induce the terminal sprouting of the remaining input as seen in vertebrates (Ironton et al., 1978)? (2) We have shown that partial denervation in anterior segments is sufficient to induce collateral sprouting. In control animals, why do the posterior abdominal segments, which often have only one cleft input, not have an increased frequency of collateral innervation? (3) By the 1st instar, we observed a high frequency of ectopic innervation not significantly different from that seen in 3rd instar larvae. In activity mutants, collateral innervation results from decreased activity in the embryo and 1st instar but not with later reduction in activity (Jarecki and Keshishian, 1995). Is there a permissive time window beyond which denervation will not induce collateral 
innervation? (4) We suggest that a cue from the target environment likely is involved in inducing sprouting. Is the signal for denervation-induced collateral sprouting attributable to direct motoneuronal interactions (for example, loss of a repulsive signal secreted by the native motoneuronal ending), or does it involve changes in the target environment itself (such as failure to downregulate a trophic factor)?

In conclusion, we have shown that Drosophila motoneurons can form novel, collateral connections in response to changes in the degree of innervation of nearby muscle fibers. Our results provide additional evidence that at least two forms of plasticity, changes in connectivity and morphology, are present in Drosophila as in vertebrate systems. In addition, we have shown that this sprouting response is restricted to the vicinity of the denervated muscle fibers, suggesting that a target-derived cue may be involved.

\section{REFERENCES}

Aigner L, Arber S, Kapfhammer JP, Laux T, Schneider C, Botteri F, Brenner H-R, Caroni P (1995) Overexpression of the neural growthassociated protein GAP-43 induces nerve sprouting in the adult nervous system of transgenic mice. Cell 83:269-278.

Atwood HL, Govind CK, Wu C-F (1993) Differential ultrastructure of synaptic terminals on ventral longitudinal abdominal muscles in Drosophila larvae. J Neurobiol 24:1008-1024.

Benoit P, Changeux J-P (1975) Consequences of tenotomy on the evolution of multi-innervation in developing rate soleus muscle. Brain Res 99:345-358.

Broadie KS, Bate M (1993a) Development of the embryonic neuromuscular synapse of Drosophila melanogaster. J Neurosci 13:144-66.

Broadie KS, Bate M (1993b) Innervation directs receptor synthesis and localization in Drosophila embryo synaptogenesis. Nature 361:350-353.

Brown MC, Holland RL (1979) A central role for denervated tissues in causing nerve sprouting. Nature 282:724-726.

Brown MC, Ironton R (1977) Motor neurone sprouting induced by prolonged tetrodotoxin block of nerve action potentials. Nature 265:459-461.

Brown MC, R Holland, Hopkins WG (1981) Motor nerve sprouting. Annu Rev Neurosci 4:17-42.

Brown ME, Lunn ER (1988) Mechanism of interaction between motoneurons and muscles. In: Plasticity of the neuromuscular system, pp 78-96. New York: Wiley.

Budnik V, Zhong Y, Wu C-F (1990) Morphological plasticity of motor axons in Drosophila mutants with altered excitability. J Neurosci 10:3754-68.

Campos-Ortega JA, Hartenstein V (1985) The embryonic development of Drosophila melanogaster. New York: Springer.

Cantera R, Nassel DR (1992) Segmental peptidergic innervation of abdominal targets in larval and adult dipteran insects revealed with an antiserum against leukokinin I. Cell Tissue Res 269:459-71.

Caroni P, Grandes P (1990) Nerve sprouting in innervated adult skeletal muscle induced by exposure to elevated levels of insulin-like growth factors. J Cell Biol 110:1307-1317.

Caroni P, Schneider C, Kiefer MC, Zapf J (1994) Role of muscle insulinlike growth factors in nerve sprouting: suppression of terminal sprouting in paralyzed muscle by IGF-binding protein 4. J Cell Biol 125:893-902.

Cash S, Chiba A, Keshishian H (1992) Alternate neuromuscular target selection following the loss of single muscle fibers in Drosophila. J Neurosci 12:2051-2064.

Chiba A, Hing H, Cash S, Keshishian H (1993) The growth cone choices of Drosophila motoneurons in response to muscle fiber mismatch. J Neurosci 13:714-732.

Chiba A, Snow P, Keshishian H, Hotta Y (1995) Fasciclin III as a synaptic target recognition molecule in Drosophila. Nature 374: $166-168$.

Chu-LaGraff Q, Schmid A, Leidel J, Bronner G, Jackle H, Doe CQ (1995) Huckebein specifies aspects of CNS precursor identity required for motoneuron axon pathfinding. Neuron 15:1041-1051.

Constantine-Paton M, Cline HT, Debski E (1990) Patterned activity, synaptic convergence, and the NMDA receptor in developing visual pathways. Annu Rev Neurosci 13:129-154.
Dahm LM, Landmesser LT (1988) The regulation of intramuscular nerve branching during normal development and following activity blockade. Dev Biol 130:621-644.

DeZazzo J, Tully T (1995) Dissection of memory formation: from behavioral pharmacology to molecular genetics. Trends Neurosci 18:212-218.

Evans PD (1992) Molecular studies on insect octopamine receptors. In: Comparative molecular neurobiology (Pichon Y, ed), pp 286-296. Boston: Verlag Birkhauser.

Evans PD, O'Shea M (1977) An octopaminergic neurone modulates neuromuscular transmission in the locust. Nature 270:257-259.

Funakoshi H, Belluardo N, Arenas E, Yamamoto Y, Casabona A, Persson H, Ibanez CF (1995) Muscle-derived neurotrophin-4 as an activitydependent trophic signal for adult motor neurons. Science 268:1495-1499.

Goodman CS, Shatz CJ (1993) Developmental mechanisms that generate precise patterns of neuronal connectivity. Cell 72:77-98.

Gorczyca MG, Phyllis RW, Budnik V (1994) The role of tinman, a mesodermal cell fate gene, in axon pathfinding during the development of the transverse nerve in Drosophila. Development 120:2143-2152.

Gurney ME, Yamamoto H, Kwon Y (1992) Induction of motor neuron sprouting in vivo by ciliary neurotrophic factor and basic fibroblast growth factor. J Neurosci 12:3241-3247.

Halfon M, Hashimoto C, Keshishian H (1995) The Drosophila Toll gene functions zygotically and is necessary for proper motoneuron and muscle development. Development 120:2143-2152.

Halpern ME, Chiba A, Johansen J, Keshishian H (1991) Growth cone behavior underlying the development of stereotypic synaptic connections in Drosophila embryos. J Neurosci 11:3227-3238.

Henderson CE, Huchet M, Changeux JP (1983) Denervation increases a neurite-promoting activity in extracts of skeletal muscle. Nature 302:609-612.

Hubel DH, Wiesel TN, Levay S (1977) Plasticity of ocular dominance columns in the monkey striate cortex. Philos Trans R Soc Lond [Biol] 278:377-409.

Ironton R, Brown MC, Holland RL (1978) Stimuli to intramuscular nerve growth. Brain Res 222:125-128.

Jan LY, Jan YN (1976) Properties of the larval neuromuscular junction in Drosophila melanogaster. J Physiol (Lond) 262:189-214.

Jarecki J, Keshishian H (1995) Role of neural activity during synaptogenesis in Drosophila. J Neurosci 15:8177-8190.

Jessell TM (1988) Adhesion molecules and the hierarchy of neural development. Neuron 1:3-13.

Johansen J, Halpern ME, Johansen KM, Keshishian H (1989a) Stereotypic morphology of glutamatergic synapses on identified muscle fiber cells of Drosophila larvae. J Neurosci 9:710-725.

Johansen J, Halpern ME, Keshishian H (1989b) Axonal guidance and the development of muscle fiber-specific innervation in Drosophila embryos. J Neurosci 9:4318-4332.

Kennedy TE, Tessier-Lavigne M (1995) Guidance and induction of branch formation in developing axons by target-derived diffusible factors. Curr Opin Neurobiol 5:83-90.

Kennedy TE, Serafini T, de la Torre JR, Tessier-Lavigne M (1994) Netrins are diffusible chemotropic factors for commissural axons in the embryonic spinal cord. Cell 78:425-435.

Keshishian H, Chiba A, Chang TN, Halfon M, Harkins EW, Jarecki J, Wang L, Anderson M, Cash S, Halpern ME (1993) The cellular mechanisms governing the development of synaptic connections in Drosophila melanogaster. J Neurobiol 24:767-787.

Keshishian H, Broadie K, Chiba A, Bate M (1996) The Drosophila neuromuscular junction: a model system for studying synaptic development and function. Annu Rev Neurosci 19:545-575.

Kopczynski CC, Davis GW, Goodman CS (1996) A neural Tetraspanin, encoded by late bloomer, that facilitates synapse formation. Science 271:1867-1870.

Kurdyak P, Atwood HL, Stewart BA, Wu C-F (1994) Differential physiology and morphology of motor axons to ventral longitudinal muscles in larval Drosophila. J Comp Neurol 350:463-472.

Landmesser L (1994) Axonal outgrowth and pathfinding. Prog Brain Res 103:67-73.

Luo Y, Raible D, Raper JA (1993) Collapsin: a protein in brain that induces the collapse and paralysis of neuronal growth cones. Cell 75:217-227.

Matthes DJ, Sink H, Kolodkin AL, Goodman CS (1995) Semaphorin II can function as a selective inhibitory of specific synaptic arborizations. Cell 81:631-639. 
Monastiriati M, Gorczyca M, Rapus J, Eckert M, White K, Budnik V (1995) Octopamine immunoreactivity in the fruit fly Drosophila melanogaster. J Comp Neurol 356:275-287.

Nose AQ, Mahajan VB, Goodman CS (1992) Connectin: a homophilic cell adhesion molecule on a subset of muscles and motoneurons that innervate them in Drosophila. Cell 70:553-567.

Nose A, Takeichi M, Goodman CS (1994) Ectopic expression of connectin reveals a repulsive function during growth cone guidance and synapse formation. Neuron 13:525-539.

Patel NH, Snow PM, Goodman CS (1987) Characterization and cloning of fasciclin III: a glycoprotein expressed on a subset of neurons and axons pathways in Drosophila. Cell 48:975-988.

Puschel AW, Adams RH, Betz H (1995) Murine semaphorin D/collapsin is a member of a diverse gene family and creates domains inhibitory for axonal extension. Neuron 14:941-948.

Schnell L, Schneider R, Kolbeck R, Barde YA, Schwab ME (1994) Neurotrophin-3 enhances sprouting of corticospinal tract during development and after adult spinal cord lesion. Nature 367:170-173.

Shatz CJ (1990) Impulse activity and the patterning of connections during CNS development. Neuron 5:745-756.

Sink H, Whitington PM (1991a) Location and connectivity of abdominal motoneurons in the embryo and larva of Drosophila melanogaster. J Neurobiol 22:298-311.

Sink H, Whitington PM (1991b) Pathfinding in the central nervous system and periphery by identified embryonic Drosophila motor axons. Development 112:307-316.
Snow PM, Bieber AJ, Goodman CS (1989) Fasciclin III: a novel homophilic adhesion molecule in Drosophila. Cell 59:313-323.

Thompson WJ (1986) Changes in the innervation of mammalian skeletal muscle fibers during postnatal development. Trends Neurosci 9:25-28.

Tower SS (1939) The reaction of muscle to denervation. Physiol Rev 19:1-48.

Vrbova G, Gordon T, Jones R (1995) Nerve-muscle interaction. London: Chapman \& Hall.

Wang J, Renger JJ, Griffith LC, Greenspan RJ, Wu C-F (1994) Concomitant alterations of physiological and developmental plasticity in Drosophila CaM Kinase II-inhibited synapses. J Neurosci 13:1373-1384.

Watson WE (1969) The response of motor neurones to intramuscular injection of botulinum toxin. J Physiol (Lond) 202:611-630.

Yin JCP, Wallach JS, Del Vecchio M, Wilder EL, Zhou H, Quinn WG, Tully T (1994) Induction of a dominant negative CREB transgene specifically blocks long-term memory in Drosophila. Cell 79:49-58.

Yin JCP, Del Vecchio M, Zhou H, Tully T (1995) CREB as a memory modulator: induced expression of a dCREB2 activator isoform enhances long-term memory in Drosophila. Cell 81:107-115.

Zhong Y, Budnik V, Wu C-F (1992) Synaptic plasticity in Drosophila memory and hyperexcitable mutants: role of cAMP cascade. J Neurosci 12:644-651.

Zhong Y (1995) Mediation of PACAP-like neuropeptide transmission by coactivation of Ras/Raf and cAMP signal transduction pathways in Drosophila. Nature 375:588-592. 